\title{
Wireless Addressable Cortical Microstimulators Powered by Near-infrared Harvesting
}

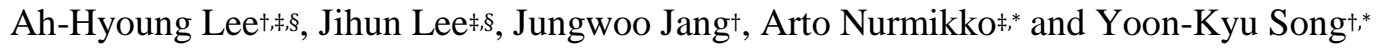 \\ ${ }^{\dagger}$ Graduate School of Convergence Science and Technology, Seoul National University, Seoul 08826, \\ Republic of Korea \\ ¥School of Engineering, Brown University, Providence, RI 02912, USA \\ $\S$ These authors contributed equally to this work. \\ *To whom correspondence should be addressed. e-mail: songyk@snu.ac.kr; arto_nurmikko@brown.edu
}

\section{Supporting Information Table of Contents}

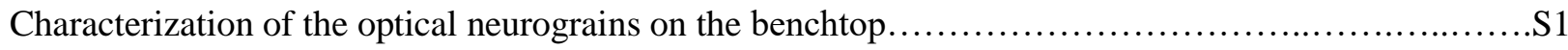

Pre- and post-stimulus spike counts evoked by single-pulse stimulation of the optical neurograin......S2-S3 A continuous high-frequency stimulation waveform injected by the optical neurograin..................S4

Neural responses evoked by burst-mode intracortical stimulation of the optical neurograin..........S5-S7

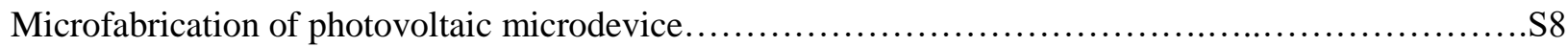

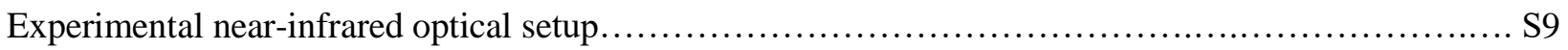

Neural data processing and stimulation artifact removal.......................................... 10

Future miniaturization techniques for the microstimulator device $\ldots \ldots \ldots \ldots \ldots \ldots \ldots \ldots \ldots \ldots \ldots \ldots \ldots \ldots \ldots \ldots \ldots$ 
a

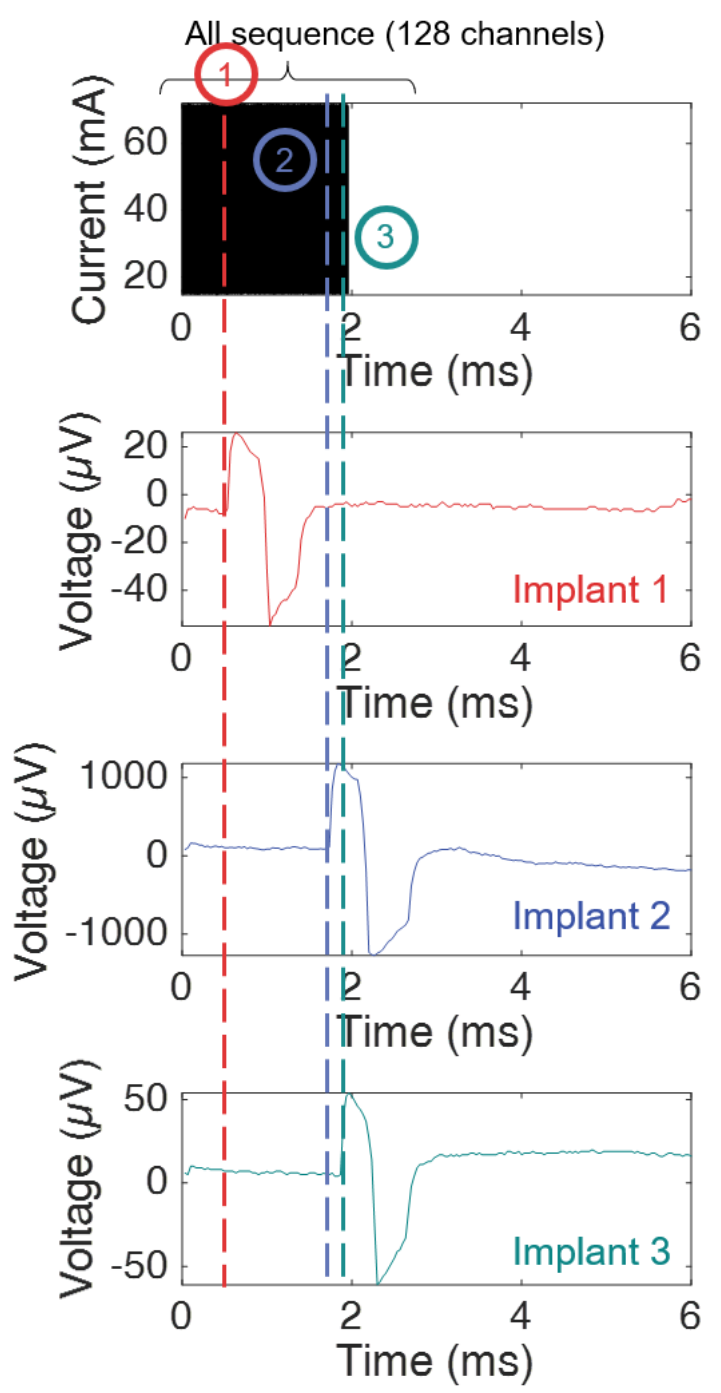

b
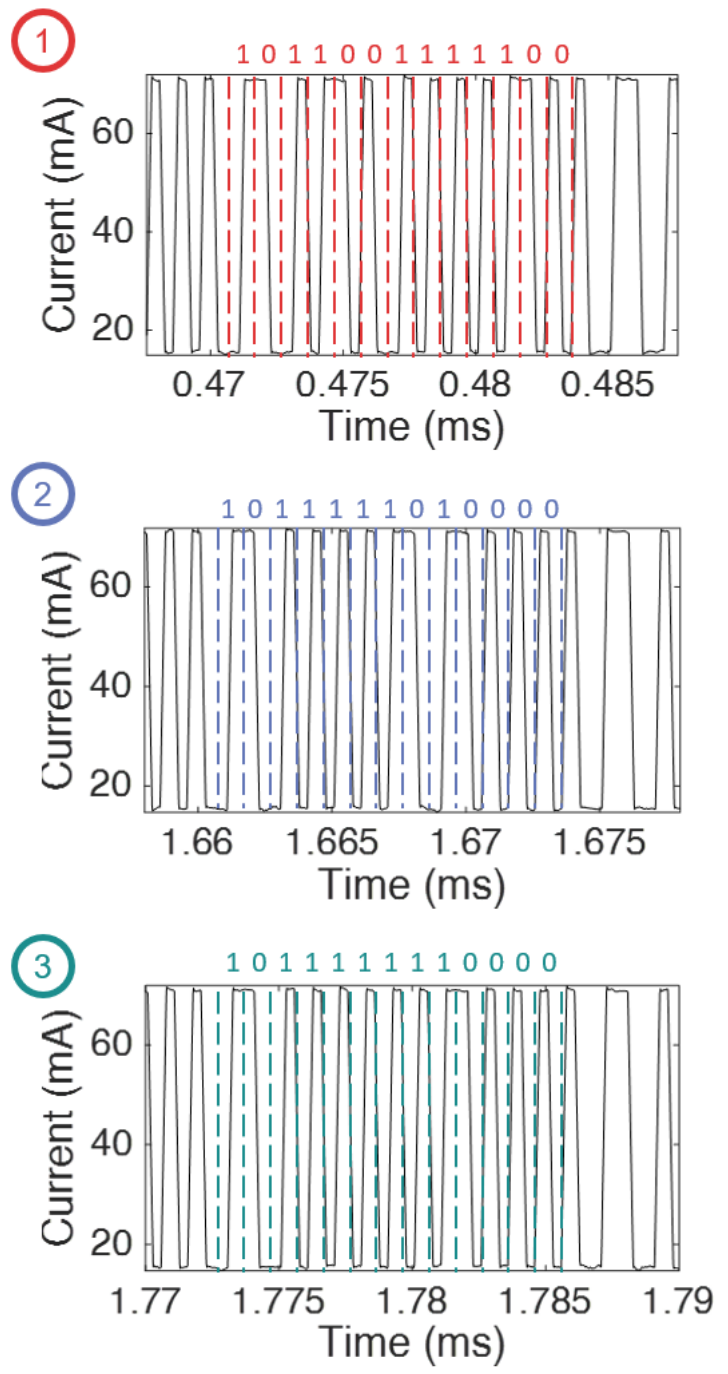

Figure S1. Demonstration of Time-division multiplexing (TDM) of optical neurograins on the benchtop. (a) The input current to the laser diode carrying the 1 Mbps downlink (top) and the timing-controlled stimulation waveforms injected by 3 optical neurograins and recorded in the saline. (b) Expanded views on the downlink sequences, which triggered each chip to generate a biphasic electrical stimulation. Each downlink command consists of the 4-bit header (1011), the 7-bit address, and the 2-bit pulse width command. After the laser ablation on the 7-bit metallic fuse structure, each chip carries a unique device ID (e.g. implant 1 -0011111, implant $2-1110100$, implant $3-1111100$ ). Only when a given chip recognized the address sequence which matched the device ID, the device delivered the on-command electrical microstimulation. 

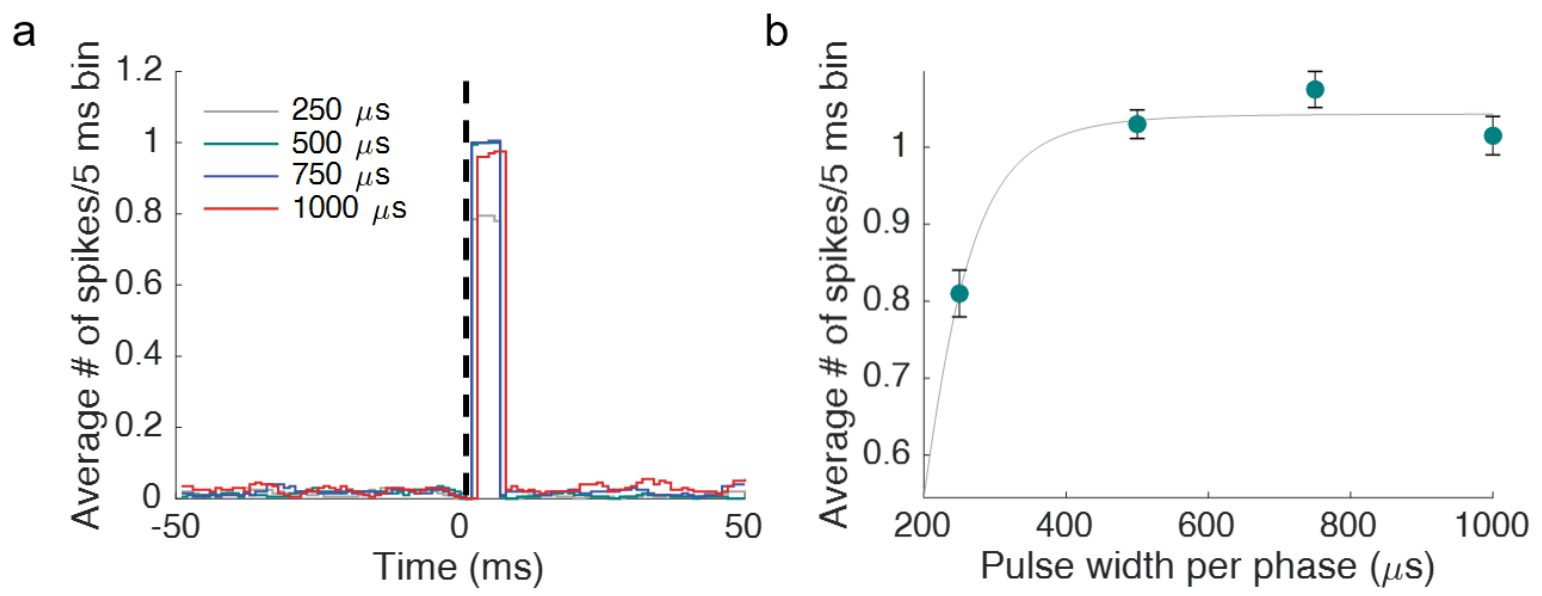

Figure S2. Pre- and post-stimulus spike counts in channel E4 evoked by optical neurograin intracortical stimulation. (a) Time histograms with a $5 \mathrm{~ms}$ binning window size and a $1 \mathrm{~ms}$ window shift showing the stimulation-generated firing response (single shot, $\mathrm{n}=200$ ). The dashed line shows the onset of the electrical stimulation ( $\mathrm{t}=0$ ). (b) The post-stimulus average number of spikes within a $20 \mathrm{~ms}$ bin, recorded under varying pulse width (per phase), showing the response saturation for long stimulus events $\sim 500 \mu \mathrm{s}$ per phase ( $n=200$ for each pulse width). Error bars denote the standard error of the mean. 


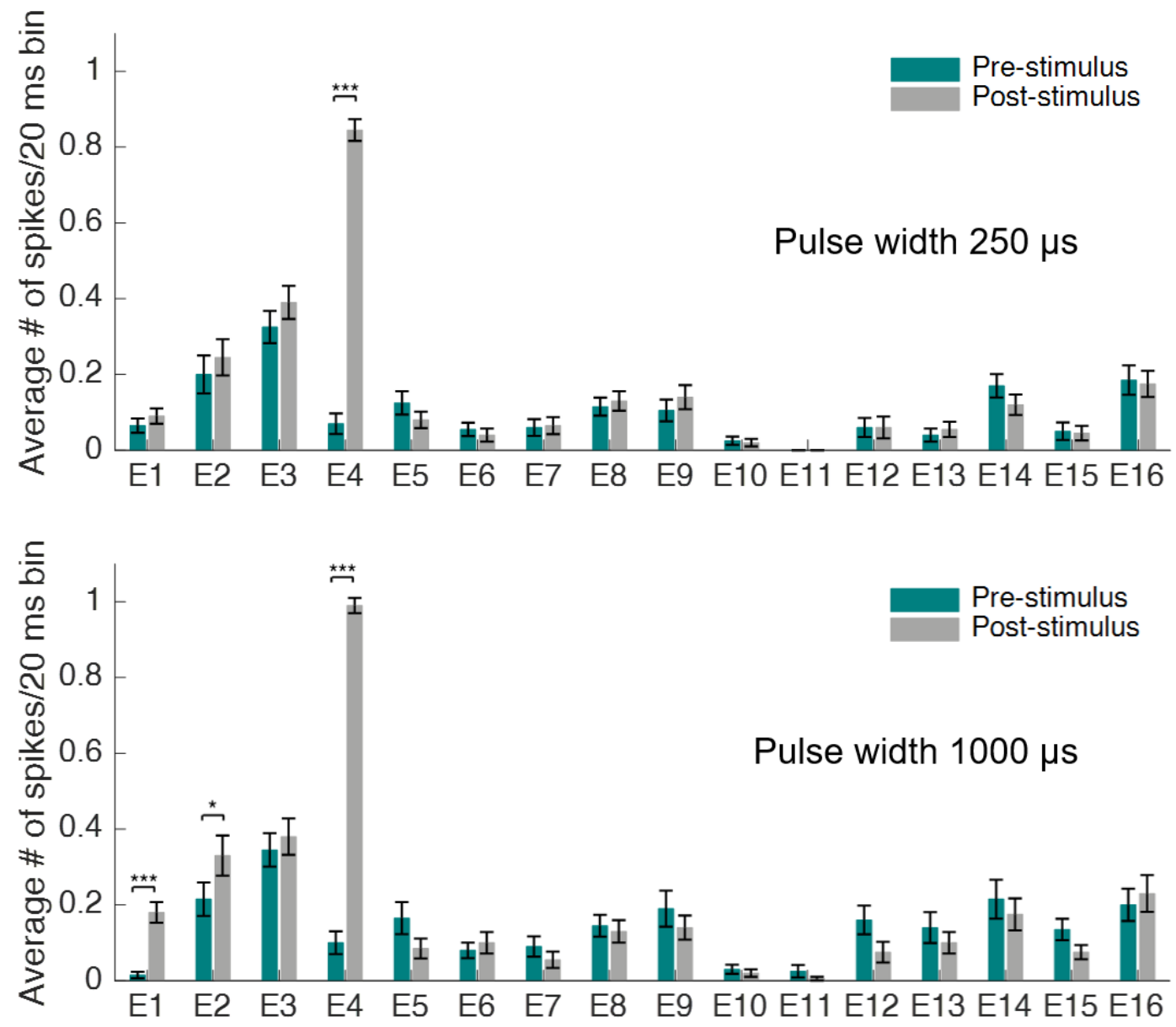

Figure S3. Pre- and post-stimulus average number of spikes per $20 \mathrm{~ms}$ bin ( $\mathrm{n}=200$, paired t-test) showing the significantly increased firing rate after the single shot electrical stimulation from the optical neurograin implant in a single channel (top, channel E4) and three channels (bottom, channel E1, E2, and E4) with the pulse width of 250 and $1000 \mu$ s per phase, respectively. Error bars denote the standard error of the mean. 

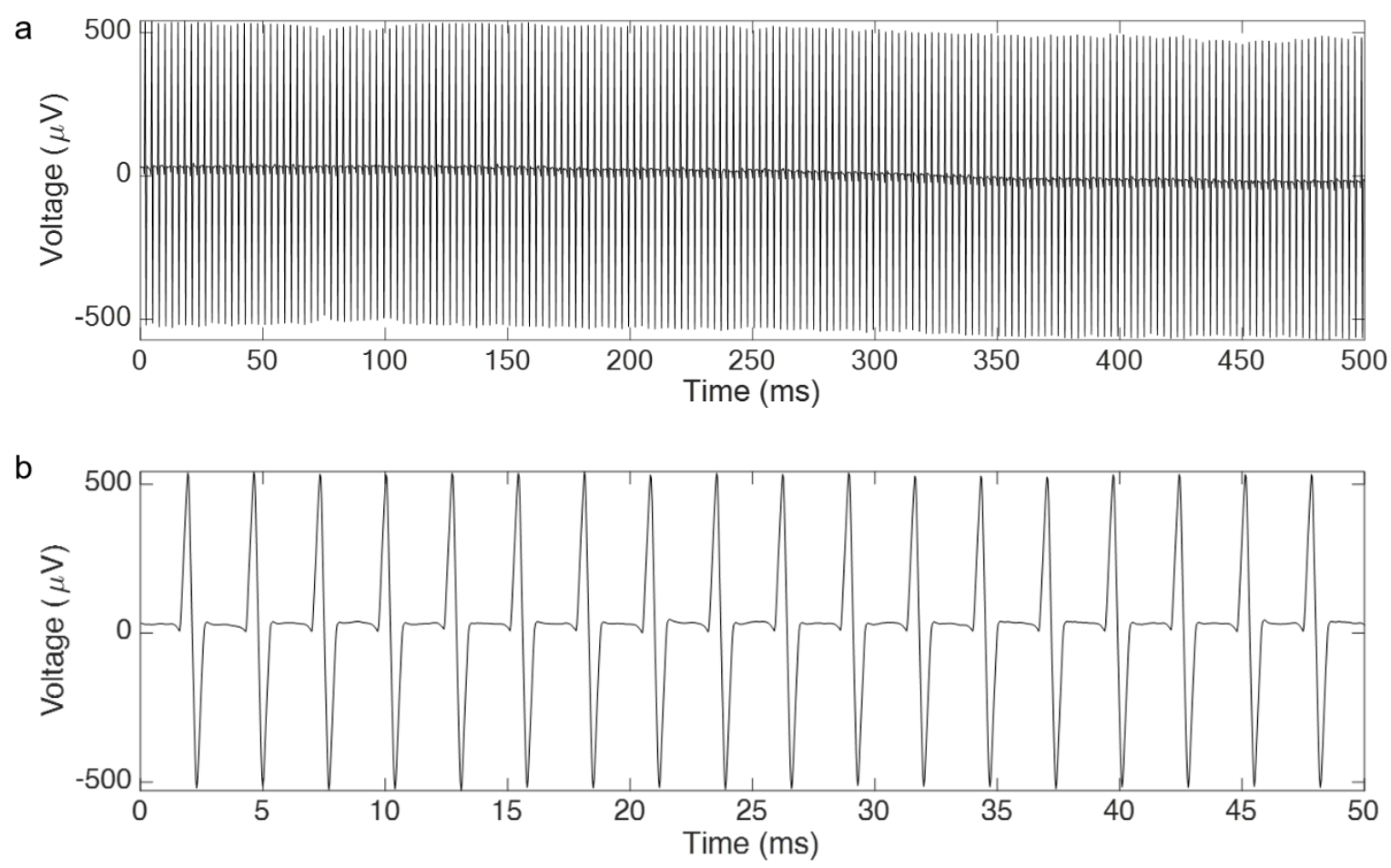

Figure S4. (a) A continuous $400 \mathrm{~Hz}$ stimulation waveform injected by the optical neurograin implant for an extended period of $500 \mathrm{~ms}$. (b) Expanded view on the waveform, which shows the achievement of a reliable downlink communication delivered by the NIR laser beam. 

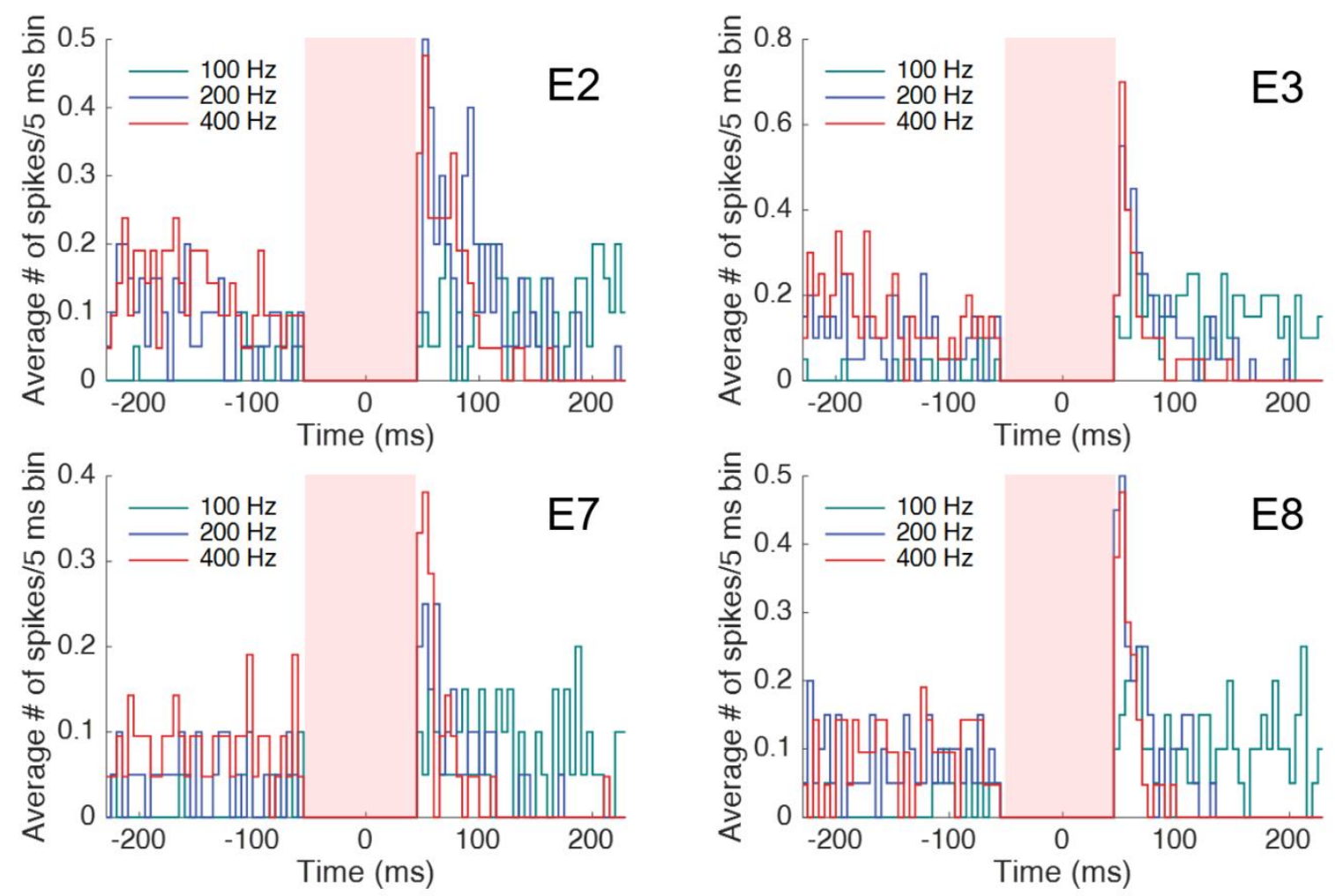

Figure S5. Spike-time histograms with $5 \mathrm{~ms}$ binning size before and after burst electrical stimulation, showing the firing response averaged across 20 trials at various stimulation frequencies (channel E2, E3, E7, and E8). The stimulation pulse width was fixed to $1000 \mu$ s per phase. The red shaded area indicates the burst stimulation period around $\mathrm{t}=0$. 


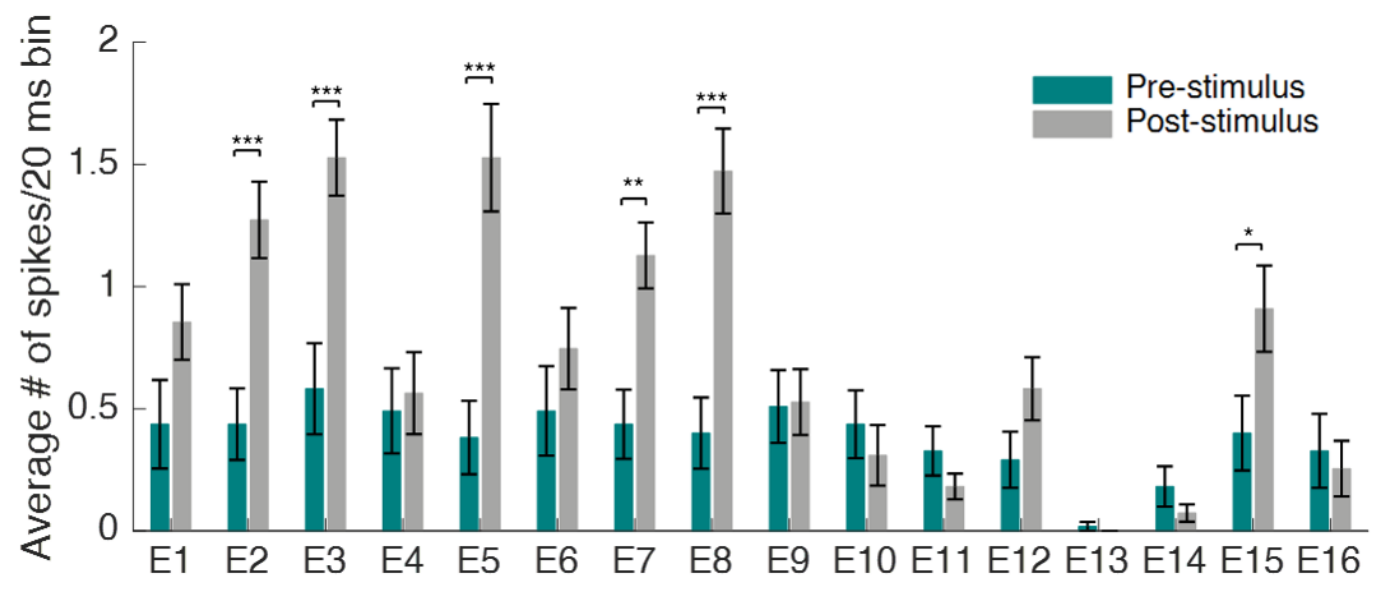

Figure S6. Pre- and post-stimulus average number of spikes per $20 \mathrm{~ms}$ bin $(\mathrm{n}=55$, paired t-test $)$ showing the significantly increased firing rate in six channels (channel E2, E3, E5, E7, E8, and E15) after the 400 $\mathrm{Hz}$ burst electrical stimulation with the pulse width of $1000 \mu$ s per phase injected by the optical neurograin. Error bars denote the standard error of the mean. 

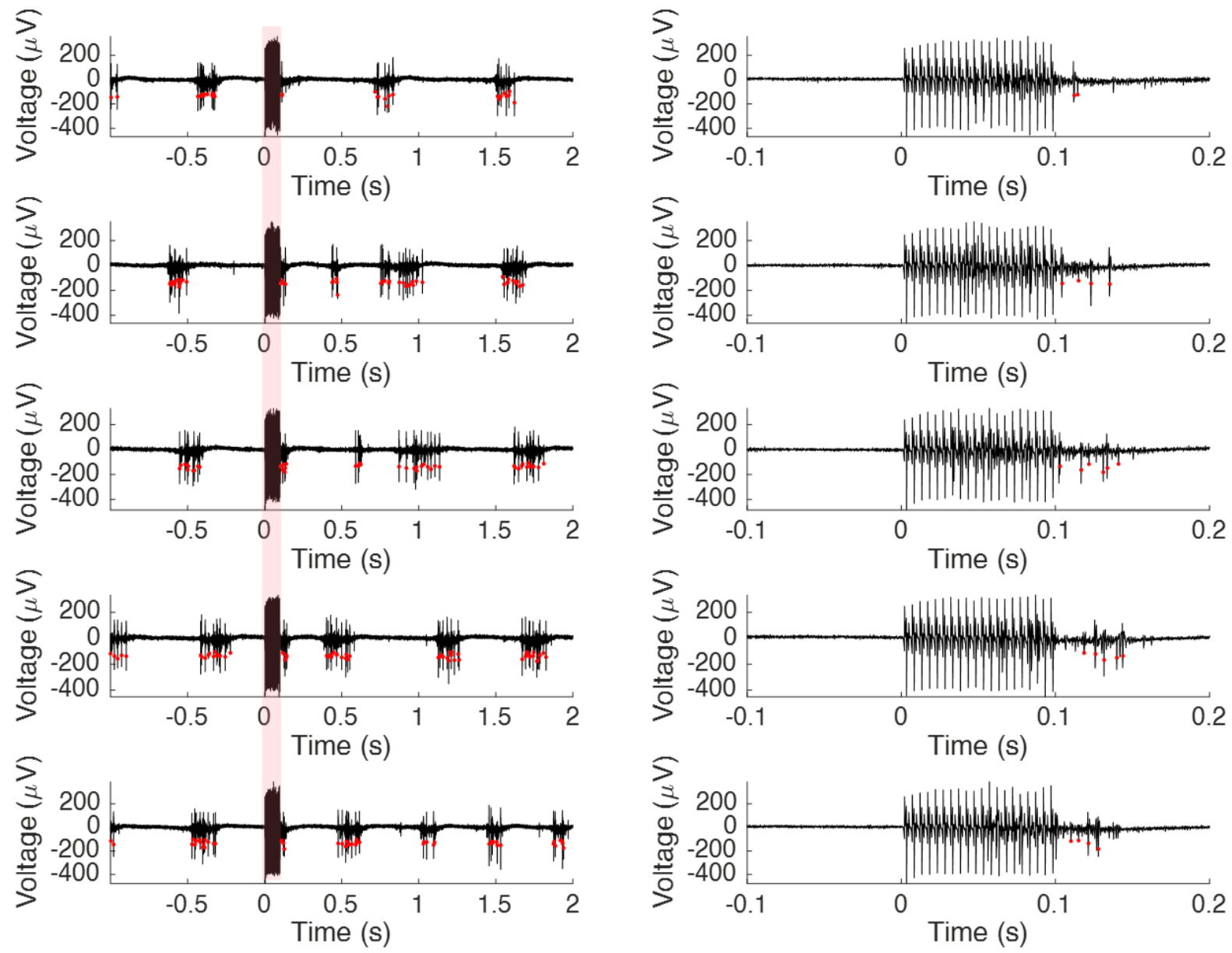

Figure S7. Spontaneous and induced burst activities recorded from the rat anesthetized by ketamine/ xylazine mixture during 5 consecutive stimulation trials (left) and expanded view on activities before and after the stimulation (right). The $400 \mathrm{~Hz}$ high-frequency electrical stimulation from optical neurograin induced the burst neural activities even right after the spontaneous burst activities, resulting in an average firing rate increase described in Figure S6. The red shaded area indicates the burst stimulation period. 
a

$\mathrm{Pd} / \mathrm{Ge} / \mathrm{Au}$ layer
$(10 / 50 / 200 \mathrm{~nm}$ thick $)$

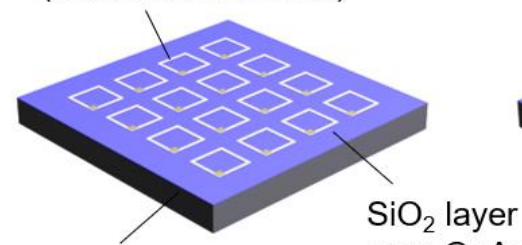

p-GaAs

Substrate on $\mathrm{n}$-GaAs top layer

(200 nm thick)

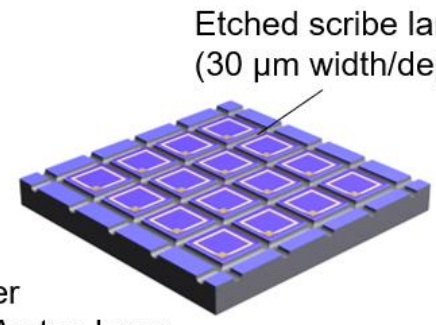

(30 $\mu \mathrm{m}$ width/depth)

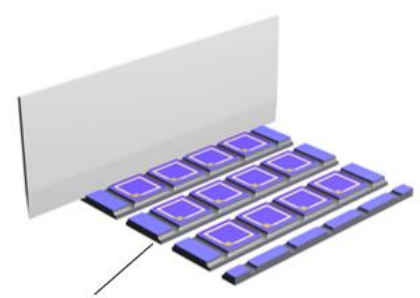

Thinned substrate (70 $\mu \mathrm{m}$ thick) b

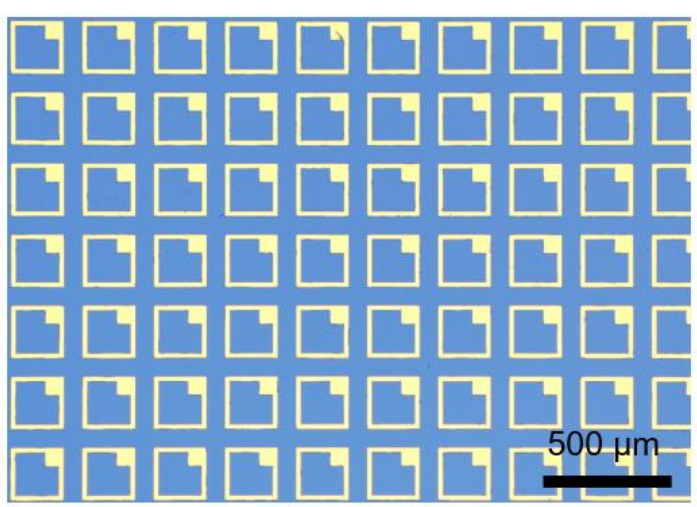

C

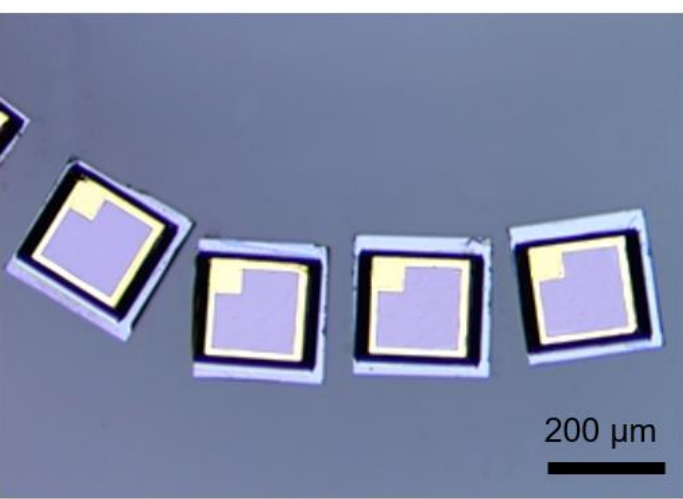

Figure S8. (a) Microfabrication steps for the miniaturized photovoltaic device in fabricating the ringshaped ohmic contact layer on n-type GaAs top layer by a sequence of photolithography using electron beam evaporation and annealing steps $\left(\mathrm{Pd} / \mathrm{Ge} / \mathrm{Au}(10 / 50 / 200 \mathrm{~nm}) ; 177^{\circ} \mathrm{C}\right.$ for $\left.5 \mathrm{~min}\right)$ (left), patterned wetetching through the $\mathrm{SiO}_{2}$ layer on the n-type top layer and $\mathrm{GaAs}$ substrate to define the scribe lanes with 30 $\mu \mathrm{m}$ width and $30 \mu \mathrm{m}$ depth (middle), substrate thinning and die separation using a razor (right). (b) Photograph of a GaAs PV wafer piece containing tens of the microfabricated PVs. (c) Photograph of GaAs PV devices with $260 \mu \mathrm{m} \times 260 \mu \mathrm{m} \times 70 \mu \mathrm{m}$ size. 


\section{Near-infrared light modulation setup}

For the experiments, we built an NIR illumination setup shown in Figure S9. The laser diode (L850P030, Thorlabs) was driven by a function generator (33522A 30MHz function/arbitrary waveform generator, Agilent) which generated the Manchester-encoded downlink waveforms at $1 \mathrm{Mbps}$. The output current from the function generator was monitored by measuring the voltage across a sense resistor (100 $\mathrm{ohm}$ ). To achieve specific timing, the function generator was controlled by an Arduino microprocessor and a push-button switch. During the in-vivo experiments, we delivered the NIR light by manually pressing on the switch while changing the downlink waveform to generate a single shot or burst of electrical stimulation. The trigger signal was also captured by an analog I/O device in the commercial neural signal processor (Ripple Neuro Nomad system; see next) used for the 32-channel MEA neural recording. The available laser diode could generate up to $5 \mathrm{~mW}$ (in the fast modulation mode). For practical reasons, we did not use widefield illumination in this work; rather an objective lens was used to focus the light on the PV microdevice.

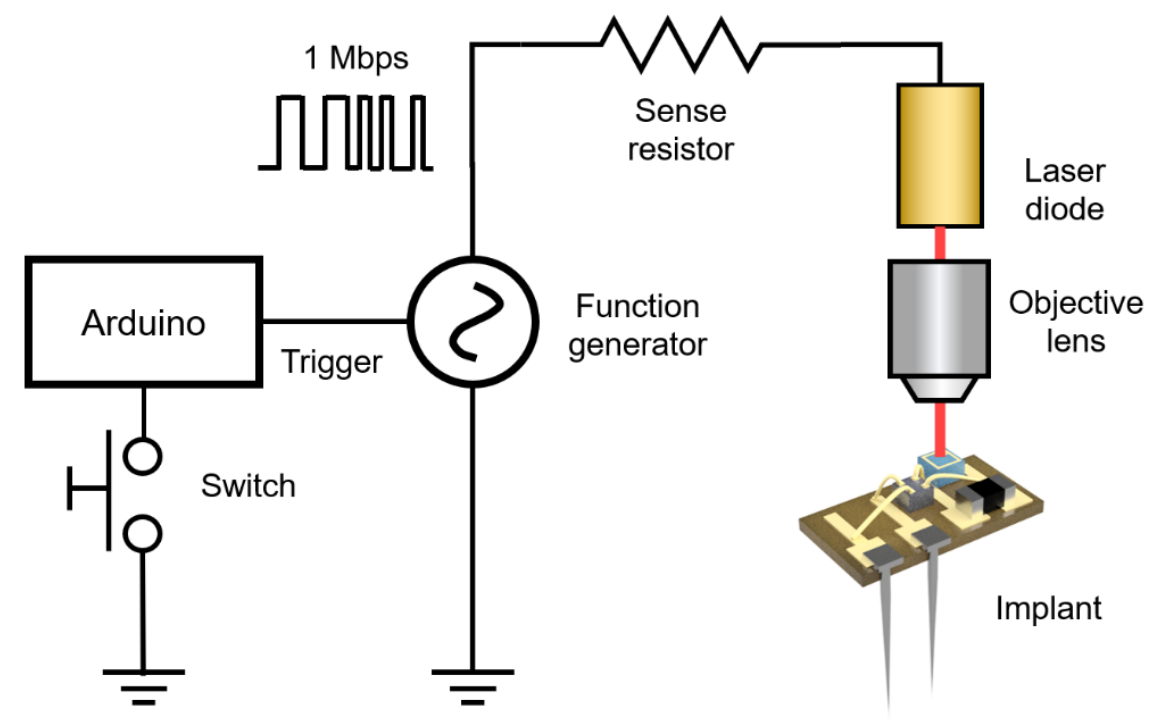

Figure S9. Block diagram of the near-infrared optical setup. An Arduino microprocessor board sent a trigger signal to a function generator activated by a manually controlled push-button switch to encode the NIR beam (encoding included the header, device ID, and user-specified stimulation parameters). A small series resistor $(100 \mathrm{ohm})$ was used to monitor the drive current into the laser diode. An objective lens focused the laser beam onto the PV element on the optical neurograin. 


\section{Neural signal processing: spike detection and stimulation artifact removal}

During the acute in-vivo experiments, we used an intracortical multielectrode array (MEA) adjacent to the ONG stimulator to record 32-channels of neural signals. The signals were delivered to a commercial 32 channel $30 \mathrm{kSa} / \mathrm{s}$ broadband recording system (Grapevine nomad, Ripple neuro). Neural signals were processed offline following the surgery, for analysis of the pre-and post-stimulus cortical spiking activity. First, we removed the $60 \mathrm{~Hz}$ noise and bandpass filtered the raw neural data with a digital finite-duration impulse response filter. We sub-selected 16 channels which showed robust spike activities across trials and removed the electrical stimulation artifacts as shown in Figure S10, including 2 ms windows immediately before and after the stimulation. We calculated unsorted multiunit spike counts across the 16 channels using a threshold-based method. Here, we used a relatively higher threshold (4.5-5 times of root-mean-square error) to prevent the false detection of spikes during the spontaneous high-frequency oscillation under ketamine/xylazine. The number of spikes before and after electrical stimulation was collected in $5 \mathrm{~ms}$ and $20 \mathrm{~ms}$ bin sizes. All results show the results from consecutive trials and no selection on trial was performed.
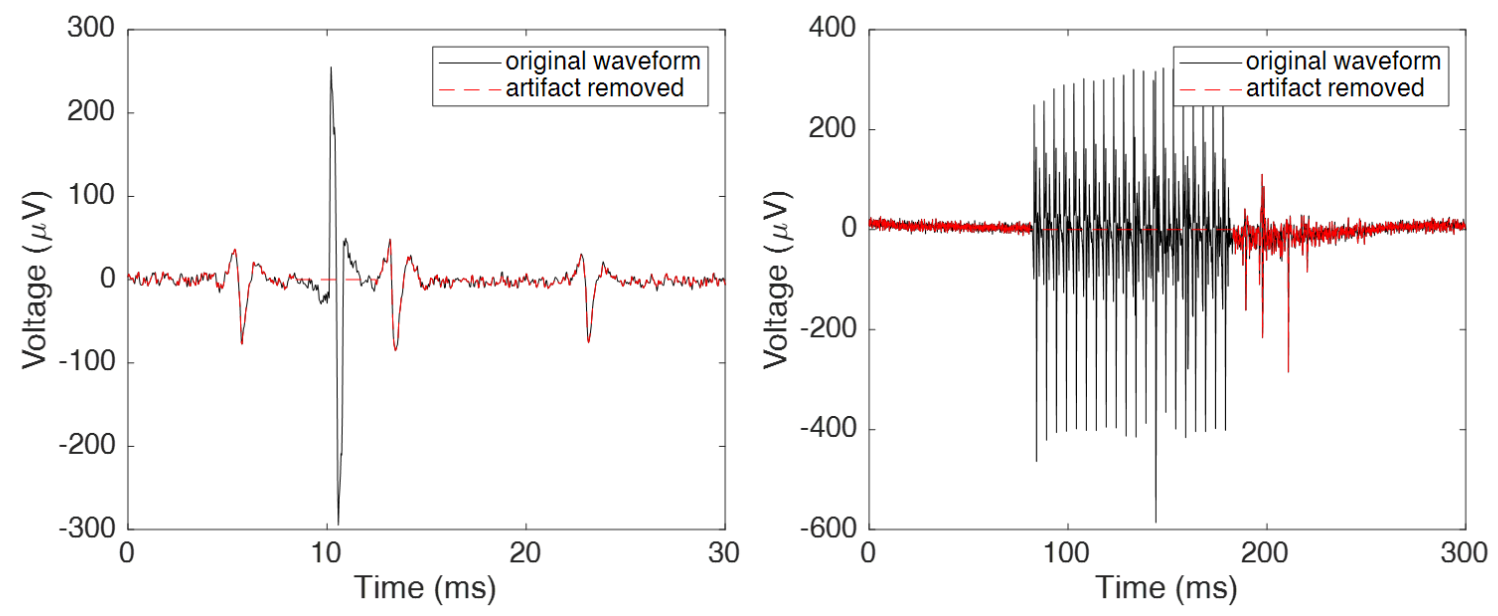

Figure S10. Process for stimulation artifact removal prior to the calculation of pre-and post-stimulus spike firing rates; single shot (left) and burst (right) stimulation. 
a

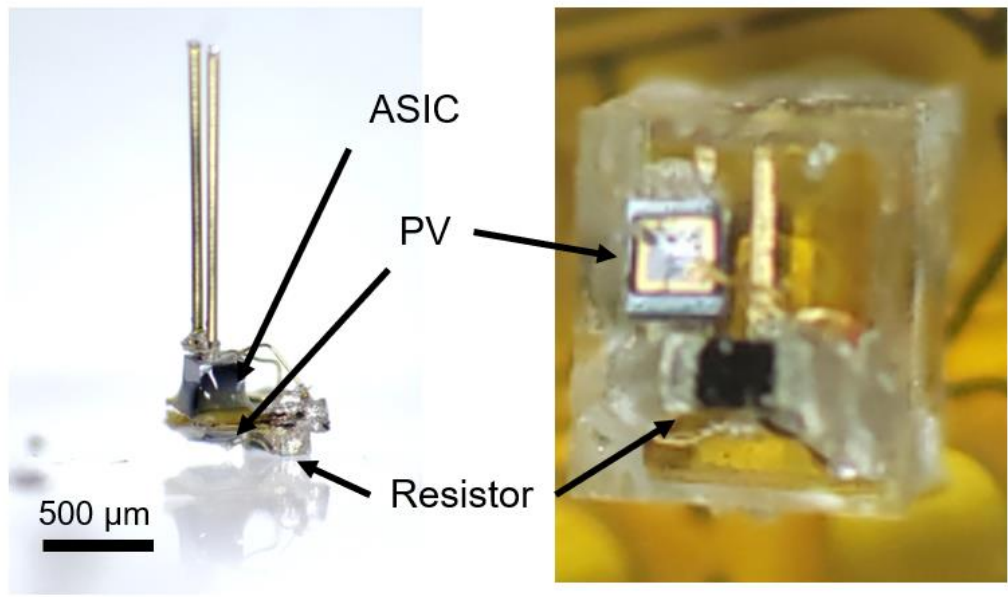

b

Wide-field NIR illumination (128 nodes)

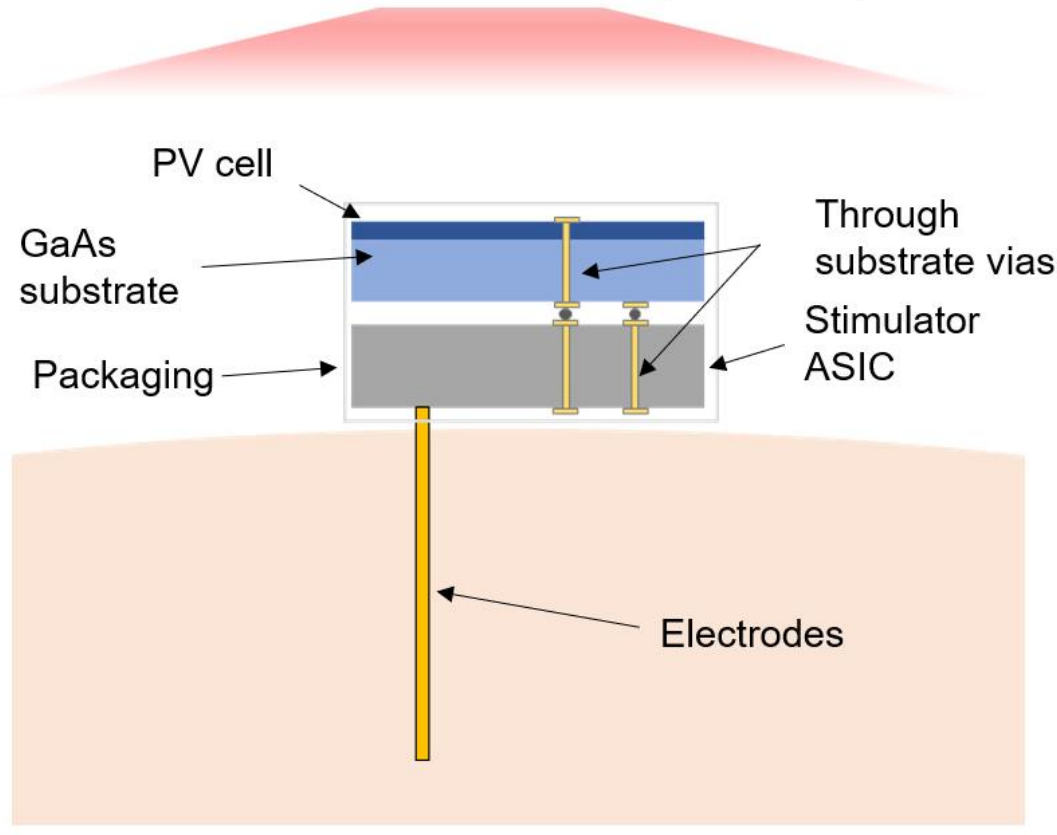

Figure S11. (a) Microphotographs of the side and top views of a fabricated benchtop mock-up device with a cross-sectional area of approximately $500 \mu \mathrm{m} \times 500 \mu \mathrm{m}$, using a piece of double-sided polyimide board to achieve a smaller footprint. (b) Conceptual illustration of a fully integrated PV cell-stimulator ASIC assembly via heterogeneous integration in a vertically stacked configuration utilizing through substrate via (TSV) connections. Since the individual microcrystal are quite small, $260 \mu \mathrm{m} \times 260 \mu \mathrm{m}$ in area, an optimal fully monolithic design of the microstimulator could reduce the overall size of the ONG down to a few hundred microns squares. 